\title{
The Role of Nanomaterials in Modulating the Structure and Function of Biomimetic Catalysts
}

\author{
Yanyan Huang ${ }^{1}$, Deshuai Yu ${ }^{2}$, Yibin Qiu ${ }^{1}$, Lanlin Chu ${ }^{1}$ and Youhui Lin ${ }^{2 *}$ \\ ${ }^{1}$ College of Light Industry and Food Engineering, Nanjing Forestry University, Nanjing, China, ${ }^{2}$ Fujian Provincial Key \\ Laboratory for Soft Functional Materials Research, Department of Physics, Research Institute for Biomimetics and Soft \\ Matter, Xiamen University, Xiamen, China
}

OPEN ACCESS

Edited by:

Zoe Pikramenou,

University of Birmingham,

United Kingdom

Reviewed by:

Xiaopeng Han,

Tianjin University, China

Guillermo Javier Copello,

Consejo Nacional de Investigaciones

Cientificas y Técnicas

(CONICET), Argentina

*Correspondence:

Youhui Lin

linyouhui@xmu.edu.cn

Specialty section:

This article was submitted to Nanoscience,

a section of the journal

Frontiers in Chemistry

Received: 30 April 2020

Accepted: 23 July 2020

Published: 29 September 2020

Citation:

Huang Y, Yu D, Qiu Y, Chu L and Lin Y

(2020) The Role of Nanomaterials in

Modulating the Structure and Function

of Biomimetic Catalysts.

Front. Chem. 8:764.

doi: 10.3389/fchem.2020.00764
Nanomaterial-incorporated enzyme mimics have so far been examined in various cases, and their properties are governed by the properties of both catalysts and materials. This review summarizes recent efforts in understanding the role of inorganic nanomaterials for modulating biomimetic catalytic performance. Firstly, the importance of enzyme mimics, and the necessity for tuning their catalysis will be outlined. Based on structural characteristics, these catalysts are divided into two types: traditional artificial enzymes, and novel nanomaterial-based enzyme mimics. Secondly, the mechanisms on how nano-sized materials interact with these catalysts will be examined. Intriguingly, incorporating various nanomaterials into biomimetic catalysts may provide a convenient and highly efficient method for the modulation of activities as well as stabilities or introduce new and attractive features. Finally, the perspectives of the main challenges and future opportunities in the areas of nanomaterial-incorporated biomimetic catalysis will be discussed. In this regard, nanomaterials as a kind of promising scaffold for tuning catalysis will attract more and more attention and be practically applied in numerous fields.

Keywords: enzyme mimic, structure and function, catalysis, nanomaterial, natural enzyme

\section{INTRODUCTION}

Owing to their excellent catalytic efficiency, unique mechanistic pathways, and complicated structural features, natural enzymes have become a tremendous source of inspiration for chemists. Numerous studies have concentrated on the simulation of their structural characteristics and functions (Hooley, 2016). So far, a variety of synthetic structures, such as porphyrins, cyclodextrins, organoselenium, and metal complexes, have been widely explored to design and construct artificial enzymes through various approaches (Dong et al., 2012; Du et al., 2017; Elemans and Nolte, 2019). In recent years, with the development of nanotechnology, many functional nanomaterials have emerged. Carbon-based, silicon-based, and metal-based nanomaterials are often endowed with properties such as large specific surface areas, easy surface modification, and high recycling efficiency. The rapid development of nanotechnology and biology has provided new opportunities for the construction of different nano-scaled structures with enzyme-like catalytic properties (Jiang et al., 2019; Liang and Yan, 2019; Wu et al., 2019). With the assistance of nanomaterials, active molecules or nanoparticles can be well-dispersed. Furthermore, bi- or multi-active components can be assembled in one nano-scaled system successfully. As a new generation of artificial enzymes, such nanocatalysts, including $\mathrm{Fe}_{3} \mathrm{O}_{4}$ (MNPs) (Gao et al., 2007), $\mathrm{CeO}_{2}$ ( $\mathrm{Xu}$ and $\mathrm{Qu}, 2014$ ), $\mathrm{V}_{2} \mathrm{O}_{5}$ (Ghosh et al., 2018), AuNPs 
(Gao et al., 2015), $\mathrm{MoS}_{2}$ nanosheets (Yin et al., 2016), graphene oxide (GO) and few-layer grapheme (Song et al., 2010), and other types of nanoparticles (Singh et al., 2017) are particularly impressive. Note: although the assembly of active components such as metal complexes and porphyrins on nanomaterial-based supports leads to the generation of nanocomposites (Wang et al., 2013; Huang Y. Y. et al., 2017), these examples treated in this review are categorized into traditional artificial enzymes, instead of nanomaterials-based artificial enzymes. The synthetic biocatalysts often possess the properties such as low cost, easy preparation, and anti-biodegradation as well as anti-denaturation when compared with natural enzymes (Huang et al., 2019). Although promising, the inherent limitations of the native forms hamper their practical applications. It is worth noting that these mimics often have relatively low catalytic performances. In this way, they can't match the high catalytic activities of natural enzymes. Furthermore, it is highly desirable that the generated enzyme mimics not only simply duplicate and mimic natural enzymes' inherent characteristics, but also may exhibit further novel properties for biological applications. Based on these unique and attractive features mentioned above, nanomaterials may also provide excellent scaffolds to the development of biomimetic catalysis for potential applications (Fan et al., 2018; Han et al., 2019, 2020).

Recently, numerous efforts have been devoted to exploring the behavior of nanomaterials in biomimetic systems (Wang et al., 2019). This review aims to provide an overview on recent developments in tuning biomimetic catalysis using nanoscale inorganic materials (Figure 1). The incorporation of nanomaterials into biomimetic catalysts provides a convenient and highly efficient method for the modulation of activities as well as the stabilities of catalysts. Alternatively, this can introduce unique and attractive features, which are not possessed by the catalysts themselves. The biomimetic catalysts discussed in this review can be roughly divided into two types according to their structural characteristics, which are traditional artificial enzymes and nanomaterials-based artificial enzymes. We hope this review may accelerate further progress in these promising areas.

\section{TRADITIONAL ARTIFICIAL ENZYMES MODULATION}

One challenge in chemistry has been the construction of synthetic systems that mimic the functions of natural enzymes. Until now, the artificial enzyme field has achieved remarkable progress. Furthermore, the incorporation of nanomaterials into these mimics have great potential for tailoring their catalytic activities and introducing other attractive features. This section will discuss nanomaterials when they interact with active molecules with enzyme-like properties.

\section{Tuning Catalysis of Active Molecules Nanomaterials for Minimizing Dimerization and Oxidative Degradation}

Metal-organic-frameworks (MOFs) and their derivatives have served as outstanding supports for heterogeneous catalysis owing to their unique features such as large specific surface areas, excellent electron transfer ability as well as rich surface chemistry. Inspired by this, a study demonstrated that metalloporphyrins could be successfully assembled with secondary binding units of metal clusters to form 2D bimetallic MOF nanosheets (Wang et al., 2016). It is well-known that porphyrin molecules easily form dimers, which may affect their catalytic activity. After self-assembling, the obtained nanomaterials could efficiently disperse the metalloporphyrins and achieve an excellent biomimetic property to catalyze the co-oxidation reaction. Based on this work, Zhang et al. further designed $\mathrm{Au}$ modified metalloporphyrinic MOF nanosheets. The obtained nanocomposites could efficiently disperse both Au nanoparticles and metalloporphyrin molecules. In this way, the nanocomposite could serve as a biomimetic catalyst for glucose $-\mathrm{H}_{2} \mathrm{O}_{2}$ cascade reaction (Huang Y. Y. et al., 2017).

\section{Nanomaterials for Increasing Binding Affinity to Substrates}

Natural enzymes have extraordinarily high catalytic efficiency. This is largely owing to their ability to bring corresponding substrates close to their active sites. Zhang et al. found that graphene oxide (GO) could dramatically increase the nucleaselike activity of the copper complexes-based DNA intercalators (Zheng et al., 2012). In their system, the copper complexes could be modified onto the surface of GO via $\pi-\pi$ interaction. The nuclease activity of the resulting conjugates was significantly higher than that of the copper complexes alone. One reason for the DNA cleavage enhancement by GO is that the obtained conjugates have a much higher binding affinity to the DNA molecules. Furthermore, another reason may be related to the generation of reactive species by accelerating the reduction of the metal center (Zheng et al., 2014).

\section{Nanomaterials for Constructing Multivalent Catalysts}

A previous review on catalysis by colloid aggregates mentioned “...groups of molecules, properly assembled, can obviously accomplish much more than an equal number of molecules functioning separately" (Menger, 1991). As the evidence began to accumulate that a lot of biological systems function via the simultaneous effects of multiple interactions, this observation was becoming increasingly important. To date, significant progress in self-assembly of catalytic-active monolayers on multivalent scaffolds, such as micelles (Dong et al., 2012), liposomes, proteins (Hou et al., 2012), and nanoparticles, has been achieved.

For example, Liu et al. used poly(amido amine) dendrimers (PD5) and cricoid proteins (SP1) as the templates to bind with superoxide dismutase (SOD) and glutathione peroxidase (GPx) catalytic centers, respectively (Figure 2A). The obtained MnPD5 and SeSP1 were then self-assembled to form dualenzyme cooperative nanowires. This nanocomposite could exhibit excellent SOD- and GPx-like properties to remove harmful reactive oxygen species (ROS) (Sun et al., 2015).

\section{Introducing Additionally New and Attractive Features}

By incorporating functional nanomaterial into enzyme mimics, such biomimetic catalysts cannot only mimic the functions of 


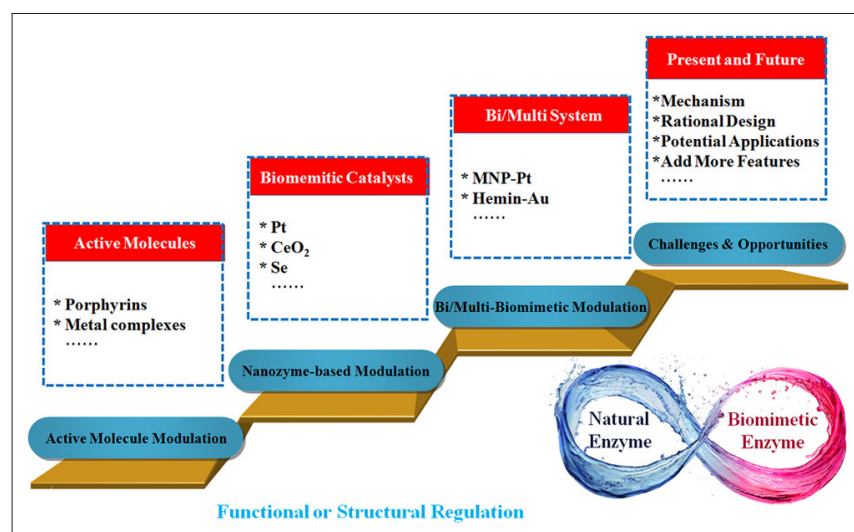

FIGURE 1 | Scheme describing the incorporation of nanomaterials into artificial enzymes for tuning catalysis.

natural enzymes, but also possess additionally advanced features in some cases.

\section{Assembling an Electrode by Binding Active Sites to Nanomaterials}

The interconversion of water and hydrogen in unitized regenerative fuel cells is considered as one important energy storage method to eliminate the temporal fluctuations of wind power and solar power. Nevertheless, to enable this technology to be economically viable, replacing current commercial platinum catalysts with cheaper and more abundant materials is highly desirable and challenging (Bashyam and Zelenay, 2006). A competitive alternative can be found in microorganisms which can metabolize molecular hydrogen using hydrogenases. Inspired by this, nanomaterials with a hydrogenase-like property can be modified on the electrodes for catalyzing this interconversion. Artero et al. prepared a noble metal-free catalytic nanomaterial through assembling a nickel bisdiphosphine-based complex and multiwalled carbon nanotubes (MWNTs). The obtained nanocomposites could serve as hydrogenase mimics. As a result, this hybrid could act as a highly specific surface area cathode material with outstanding catalytic performance even in the condition of strong acid solutions (Goff et al., 2009).

\section{Near-Infrared Photothermal Control}

$\mathrm{Qu}$ et al. demonstrated an approach to construct a $\left[\mathrm{Fe}_{2} \mathrm{~L}_{3}\right]^{4+} / \mathrm{GO}-\mathrm{COOH}-$ based peroxidase mimic. Since GO$\mathrm{COOH}$ had strong absorption in near infrared regions, this peroxidase mimic had good sensitivity to NIR and high photothermal conversion efficiency (Figure 2B). Furthermore, the integration of this nanocomposite with glucose oxidase $(\mathrm{GOx})$ could enable the creation of a catalytic ensemble for a cascade reaction. In addition, within a temperature range from 15 to $37^{\circ} \mathrm{C}, \mathrm{GOx}$ and $\left[\mathrm{Fe}_{2} \mathrm{~L}_{3}\right]^{4+}{ }_{-} \mathrm{GO}-\mathrm{COOH}$ had a higher activity at an elevated temperature. In the presence of NIR laser irradiation, an obvious absorbance band at $417 \mathrm{~nm}$ was achieved, indicating that the oxidized 2,2'-azino-bis(3-ethylbenzothiazoline-6sulfonic acid) $\left(\mathrm{ABTS}^{+}\right)$was formed. Based on NIR photothermal effect and temperature-dependent activity, the activity of a GOx$\left[\mathrm{Fe}_{2} \mathrm{~L}_{3}\right]^{4+}{ }_{-} \mathrm{GO}-\mathrm{COOH}$ cascade system could be controlled by near infrared (NIR) light (Xu et al., 2014). Taken together, based on the unique advantages of nanomaterials, they can serve as regulators to modulate the catalytic activities of active molecules with enzyme-like properties.

\section{MODULATION OF NANOMATERIAL-BASED ARTIFICIAL ENZYMES}

So far, a variety of nano-scaled materials have been discovered to have unique enzyme-like activities (Wu et al., 2019). Additionally, a few "small molecule" systems, such as deoxyribonucleic acid and ionic liquid, have shown the ability to modulate the activity of these novel enzyme mimics. In this section, as an alternative to small molecule inhibition, recent progress in nanomaterials provide a novel pathway to regulate the catalytic behaviors of nanomaterial-based artificial enzymes.

\section{Regulation of Stability and Activity of Nanomaterials-Based Artificial Enzymes}

The rapid advances in solid-supported catalysts prompt scientists to examine whether matrices can promote the catalytic behavior of these nano-sized enzyme mimics. Compared with bulk materials, materials filled in the nanochannels often exhibit superior performances such as enhanced catalytic activities and improved stabilities. Ling and Gao's group constructed combined $\mathrm{Fe}$ porphyrin and $\mathrm{Zr}^{4+}$ ions within MOFs. The obtained nanomaterials were noted as $\mathrm{PMOF}(\mathrm{Fe})$ and further assembled with ultrasmall Pt nanoparticles to generate Pt@PMOF(Fe). The MOFs could efficiently hinder the aggregation of the Pt component. In this way, the nanocomposite exhibited high peroxidase-like property and stability (Ling et al., 2020).

In addition, $\mathrm{Qu}$ et al. demonstrated one rational method for constructing $\mathrm{CeO}_{2} \mathrm{NPs}$ encapsulated on porous carbonaceous frameworks (Figure 2C). The porous carbonaceous frameworks could promote the formation of a high degree of very small, welldispersed, and stable $\mathrm{CeO}_{2}$ NPs. The obtained nanocomposites exhibited excellent oxidase-like ability compared with other types of $\mathrm{CeO}_{2}$ NPs (Cao et al., 2018). Recently, they used $\mathrm{GO}$ as the template to assemble selenium nanoparticles. The formed GO-Se nanocomposites could serve as GPx mimics to eliminate harmful $\mathrm{H}_{2} \mathrm{O}_{2}$ with the assistance of glutathione. This enzyme mimic performed enhanced enzyme-like activities in comparison to their independent components and exhibited potential antioxidant effect for cytoprotection (Huang Y. Y. et al., 2017). Besides the mentioned materials, a carbon nanotube can also be used as a support for regulating the stability and activity of nanomaterial-based artificial enzymes.

\section{Forming Hybrid Nanomaterials for Artificial Cascade Systems}

In addition to regulating catalysis, nano-sized enzyme mimics can also be used for constructing artificial enzymatic cascade systems. For example, $\mathrm{Qu}$ et al. used polydopamine (pDA) as the bridge for assembling $\mathrm{V}_{2} \mathrm{O}_{5}$ nanowires and $\mathrm{MnO}_{2}$ 


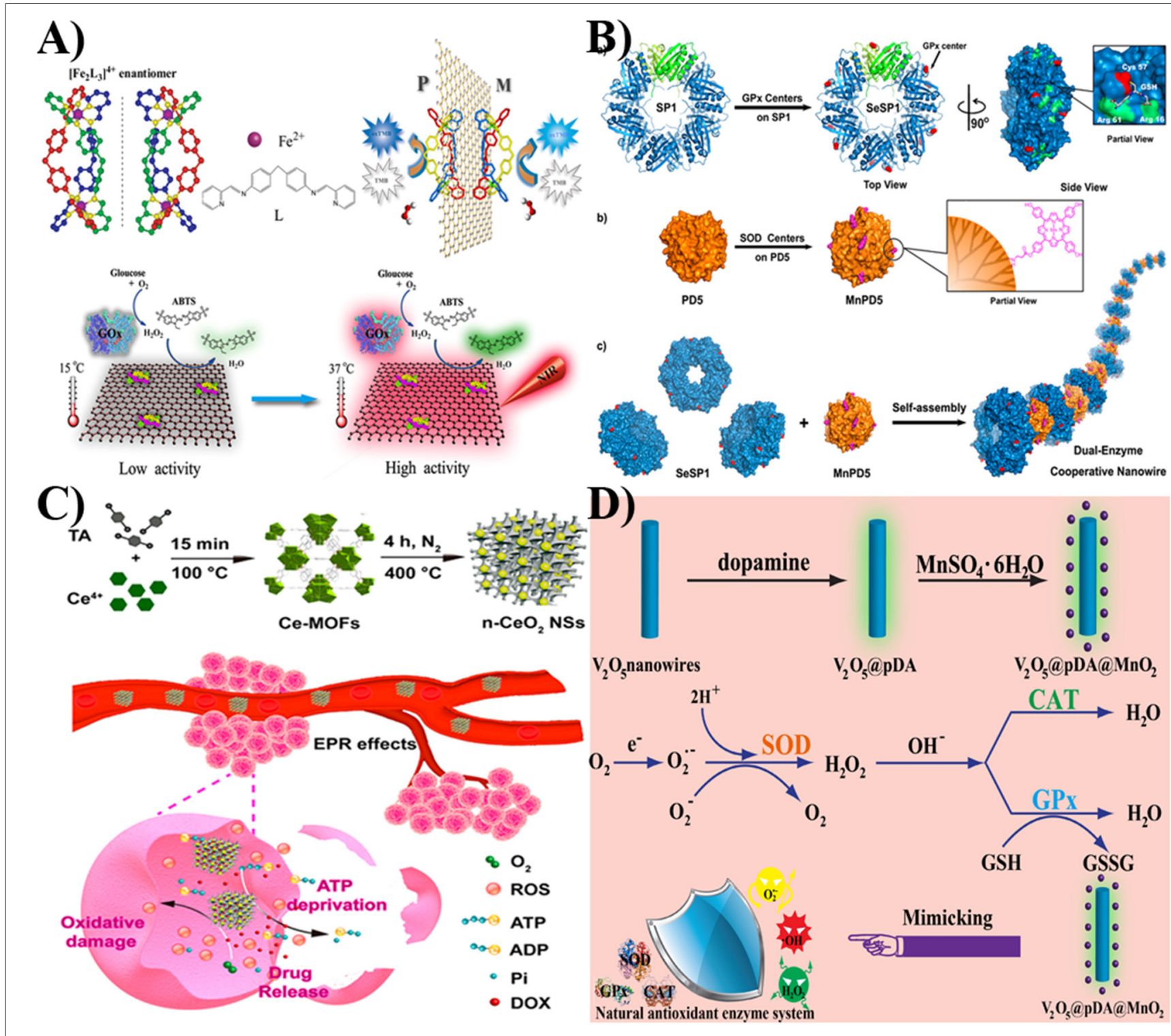

FIGURE 2 | (A) The peroxidase-like property of $\left[\mathrm{Fe}_{2} \mathrm{~L}_{3}\right]^{4+} / \mathrm{GO}-\mathrm{COOH}$ can be controlled by NIR. Reprinted with permission from Xu et al. Copyright (2014) Wiley-VCH. (B) The assembly of SeSP1 and MnPD5 to form dual-enzyme cooperative nanowire. Reprinted with permission from Sun et al. Copyright (2015) American Chemical Society. (C) The design of the $\mathrm{CeO}_{2}$ NPs encapsulated-porous carbonaceous frameworks for cancer therapy. Reprinted with permission from Cao et al. Copyright (2018) American Chemical Society. (D) The assembly and the scheme of the $\mathrm{V}_{2} \mathrm{O}_{5} @ \mathrm{pDA} @ \mathrm{MnO}_{2}$ nanozyme for mimicking natural antioxidant enzyme system. Reprinted with permission from Huang et al. Copyright (2016) Wiley-VCH.

nanoparticles (Figure 2D). In their work, the $\mathrm{MnO}_{2}$ component could serve as SOD mimics to transform a superoxide radical to $\mathrm{H}_{2} \mathrm{O}_{2}$ and $\mathrm{O}_{2}$. With the inherent catalase-like property of $\mathrm{MnO}_{2}$ and the GPx-like ability of the $\mathrm{V}_{2} \mathrm{O}_{5}$ component, the generated $\mathrm{H}_{2} \mathrm{O}_{2}$ would be scavenged as harmless products. With the antioxidant enzyme-like properties of nanozymes and the antioxidant ability of pDA, the nanocomposites could effectively scavenge overexpressed ROS and protect intracellular components against oxidative damage. This $\mathrm{V}_{2} \mathrm{O}_{5} @ \mathrm{pDA} @ \mathrm{MnO}_{2}$ nanozyme could mimic intracellular antioxidant enzyme-based defense systems for cytoprotection. Further animal inflammatory models illustrated that the nanocomposites could serve as potential nanoagents for ameliorating inflammation (Huang et al., 2016). Owing to their advantages of large specific surface area, easy surface modification, and excellent electron transfer ability, nanomaterials have been used as promising regulators to control the catalytic properties of artificial enzymes.

\section{MODULATION OF MULTIPLE BIO- OR/AND BIOMIMETIC CATALYSTS}

The attachment of different bio- or/and biomimetic catalysts on the same carbon nanomaterial provides a simple pathway 
for fabricating catalytic ensembles which possess synergic and complementary properties. Besides porous silica structures, mesoporous carbon can serve as a support for the construction of nanostructured multi-catalyst systems. For instance, combining MNPs with Pt nanoparticles in ordered mesoporous carbon has been prepared by Park et al. (Kim et al., 2014). As a result, this composite exhibited the enhanced peroxidase-like activity in comparison to independent MNPs, which could be due to the synergetic effect.

In addition to traditional graphene-based (Xue et al., 2014) and porous-based materials (He et al., 2013), graphenemesoporous silica hybrid as a novel nanomaterial can be used for bio- or multi-artificial catalyst modulation. Very recently, $\mathrm{Qu}$ and Ren's group reported that hemin and AuNPs with complementary functions could be located in different regions in a graphene-mesoporous silica hybrid. Firstly, through weak $\pi-\pi$ stacking interactions, hemin was tethered on the exposed surface of graphene. Since graphene could hinder the selfdimerization of hemin molecules, the as-prepared hemincontained nanoconjugates can act as a highly efficient peroxidase mimic. Then, AuNPs, with glucose oxidase-mimicking activity, can be formed on the $\mathrm{NH}_{2}$ groups functional silica surface by in-situ reduction. Consequently, these integrated catalysts exhibit glucose oxidase-like and peroxidase-like catalytic activities. More importantly, these nanocomplexes with multiple catalytic sites are able to catalyze artificial cascade reactions, without the addition of natural enzymes. This finding might pay the way to anchor multiple enzyme mimics on solid matrices for multicomponent cascade transformation or realizing artificial organelles in the future (Lin et al., 2015). In addition to regulating the activity of active molecules or biomimetic catalysts, nanomaterials can also be used for the regulation of multibiomimetic systems.

\section{CHALLENGES AND FUTURE OPPORTUNITIES}

Although remarkable progress has been made, the development of nanomaterials incorporating enzymes and artificial enzymes is still in a relatively early stage. In order to carry out further research in these mentioned areas, the following challenges need to be addressed:

1) The mechanism leading to the change of catalytic performance. Obvious changes in activity, specificity, or selectivity of these catalysts may appear when combining artificial enzymes with various nanomaterials. However, in many cases, the mechanism on how nanoscale materials affect the properties of these catalysts is not properly understood and validated.

\section{REFERENCES}

Bashyam, R., and Zelenay, P. (2006). A class of non-precious metal composite catalysts for fuel cells. Nature 443, 63-66. doi: 10.1038/nature05118
2) Rational design of surface functionalized nano-sized materials. It is important to take into account surface properties in the interactions of nanomaterials with bio- and biomimetic catalysts.

3) Apart from the surface properties, the size, morphology as well as composition of nanomaterials also play a crucial role. This point needs systematical examination in the future.

4) Constructing novel integrated catalysts which possess superior and often unique functions for their practical applications is still in its infancy. More attention should be devoted to the use of functional nanomaterials for constructing catalysts with new properties.

5) Although many enzymes and artificial enzymes have been combined with nanomaterials, it is indeed necessary to further investigate the potential applications of nanomaterials in tuning other biomimetic reactions.

6) It is important to further investigate their potential industrial applications.

\section{CONCLUSIONS}

Enzymes have attracted scientists' curiosity and attention for a long time. The concepts of enzyme-catalyzed transformations have been a tremendous source of inspiration for fabricating synthetic catalysts which possess the ability of mimicking the essential or general properties of natural enzymes. Particularly, recent developments in nanotechnology have enhanced the possibility for assembling engineered nanomaterials with biomimetic catalysts. This review systematically summarized the latest developing progress of nanomaterials especially inorganic nanomaterials in regulating biological and biomimetic catalysis. These works have exhibited great potential for applications ranging from the control and regulation of activity and biosensing to the separation and construction of hybrid nanoarchitectures. Evidentially, breakthroughs in biotechnology, nanotechnology as well as bionic technology may pave the way for constructing novel hybrid structures for broad applications by overcoming the unresolved issues and challenges.

\section{AUTHOR CONTRIBUTIONS}

YH, DY, YQ, LC, and YL conceived and wrote this paper. All the authors read and approved the final manuscript.

\section{FUNDING}

This work was financially supported by AcRF Tier 1(R-143-000497-112), National Nature Science Foundation (Nos. 21271040, 51073031), and the Excellent Young Teachers Training.

Cao, F. F., Zhang, Y., Sun, Y. H., Wang, Z. Z., Zhang, L., Huang, Y. Y., et al. (2018). Ultrasmall nanozymes isolated within porous carbonaceous frameworks for synergistic cancer therapy: enhanced oxidative damage and reduced energy supply. Chem. Mater. 30, 7831-7839. doi: 10.1021/acs.chemmater.8b03348 
Dong, Z. Y., Luo, Q., and Liu, J. Q. (2012). Artificial enzymes based on supramolecular scaffolds. Chem. Soc. Rev. 41, 7890-7908. doi: $10.1039 / \mathrm{c} 2 \operatorname{cs} 35207 \mathrm{a}$

Du, B. J., Li, D., Wang, J., and Wang, E. K. (2017). Designing metal-contained enzyme mimics for prodrug activation. Adv. Drug Delivery Rev.118, 78-93. doi: 10.1016/j.addr.2017.04.002

Elemans, J. A. A. W., and Nolte, R. J. M. (2019). Porphyrin cage compounds based on glycoluril-from enzyme mimics to functional molecular machines. Chem. Commun. 55, 9590-9605. doi: 10.1039/C9CC04372A

Fan, K. L., Xi, J. Q., Fan, L., Wang, P. X., Zhu, C. H., Tang, Y., et al. (2018). In vivo guiding nitrogen-doped carbon nanozyme for tumor catalytic therapy. Nat. Common. 9:1440. doi: 10.1038/s41467-018-03903-8

Gao, L., Liu, M. Q., Ma, G. F., Wang, Y. L., Zhao, L. N., Yuan, Q., et al. (2015). Peptide-Conjugated Gold Nanoprobe: Intrinsic Nanozyme-linked immunsorbant assay of integrin expression level on cell membrane. ACS Nano 9, 10979-10990. doi: 10.1021/acsnano.5b04261

Gao,. L. Z., Zhuang, J., Nie, L., Zhang, J. B., Zhang, Y., Gu, N., et al. (2007). Intrinsic peroxidase-like activity of ferromagnetic nanoparticles. Nat. Nanotechnol. 2, 577-583. doi: 10.1038/nnano.2007.260

Ghosh, S., Roy, P., Karmodak, N., Jemmis, E. D., and Mugesh, G. (2018). Nanoisozymes: crystal-facet-dependent enzyme-mimetic activity of $\mathrm{V}_{2} \mathrm{O}_{5}$ nanomaterials. Angew. Chem. Int. Ed. 57, 4510-4515. doi: 10.1002/anie.201800681

Goff, A. L., Artero, V., Jousselme, B., Tran, P. D., Guillet, N., Métayé, R., et al. (2009). From hydrogenases to noble metal-free catalytic nanomaterials for $\mathrm{H}_{2}$ production and uptake. Science 326, 1384-1387.doi: 10.1126/science.1179773

Han, X. P., Ling, X. F., Wang, Y., Ma, T. Y., Zhong, C., Hu, W. B., et al. (2020). Generationof nanoparticle, atomic-cluster, and single-atom cobalt catalysts from zeolitic imidazole frameworks by spatial isolationand their use in zinc-air batteries. Angew.Chem. Int. Ed. 58, 5359-5364. doi: 10.1002/anie.201901109

Han, X. P., Ling, X. F., Yu, D. S., Xie, D. Y., Li, L. L., Peng, S. J., et al. (2019). Atomically dispersed binary Co-Nisites in nitrogen-doped hollow carbon nanocubes for reversible oxygen reduction and evolution. Adv. Mater. 31:1905622. doi: 10.1002/adma.201905622

He, X. L., Tan, L. F., Chen, D., Wu, X. L., Ren, X. L., Zhang, Y. Q., et al. (2013). $\mathrm{Fe}_{3} \mathrm{O}_{4}$-Au@mesoporous $\mathrm{SiO}_{2}$ microspheres: an ideal artificial enzymatic cascade system. Chem. Commun. 49, 4643-4645. doi: 10.1039/c3cc40622a

Hooley, R. (2016). Biomimetic catalysis: taking on the turnover challenge. Nat. Chem. 8, 202-204. doi: 10.1038/nchem.2461

Hou, C. X., Luo, Q., Liu, J. L., Miao, L., Zhang, C. Q., Gao, Y. Z., et al. (2012). Construction of GPx active centers on natural protein nanodisk/nanotube: a new way to develop artificial nanoenzyme. ACS Nano 6, 8692-8701. doi: $10.1021 / \mathrm{nn} 302270 \mathrm{~b}$

Huang, Y. Y., Liu, C. Q., Pu, F., Liu, Z., Ren, J. S., and Qu, X. G. (2017). AGO-Se nanocomposite as an antioxidant nanozyme for cytoprotection. Chem. Commun. 53, 3082-3085. doi: 10.1039/C7CC00045F

Huang, Y. Y., Liu, Z., Liu, C. Q., Ju, E. G., Zhang, Y., Ren, J. S., et al. (2016). Self-assembly of multi-nanozymes to mimic an intracellularantioxidant defense system. Angew. Chem. Int. Ed. 55, 6646-6650. doi: 10.1002/anie.201600868

Huang, Y. Y., Ren, J. S., and Qu, X. G. (2019). Nanozymes: classification, catalytic mechanisms, activity regulation, and applications. Chem. Rev. 119, 4357-4412. doi: 10.1021/acs.chemrev.8b00672

Jiang, D. W., Ni, D. L., Rosenkrans, Z. T., Huang, P., Yan, X. Y., and Cai, W. B. (2019). Nanozyme: new horizons for responsive biomedical applications. Chem. Soc. Rev. 48, 3683-3704. doi: 10.1039/C8CS00718G

Kim, M. I., Ye, Y., Woo, M. A., Lee, J., and Park, H. G. (2014). A highly efficient colorimetric immunoassay using a nanocomposite entrapping magnetic and platinum nanoparticles in ordered mesoporous carbon. Adv. Healthc. Mater. 3, 36-41. doi: 10.1002/adhm.201300100

Liang, M. M., and Yan, X. Y. (2019). Nanozymes: from new concepts, mechanisms, and standards to applications. Acc. Chem. Res. 52, 2190-2200. doi: 10.1021/acs.accounts.9b00140

Lin, Y. H., Wu, L., Huang, Y. Y., Ren, J. S., and Qu, X. G. (2015). Positional assembly of hemin and gold nanoparticles in graphene-mesoporous silica nanohybrids for tandem catalysis. Chem. Sci. 6, 1272-1276. doi: $10.1039 / \mathrm{C} 4 \mathrm{SC} 02714 \mathrm{~K}$

Ling, P. H., Cheng, S., Chen, N., Qian, C. H., and Gao, F. (2020). Nanozymemodified metal-organic frameworks with multienzymes activity as biomimetic catalysts and electrocatalytic interfaces. ACS Appl. Mater. Interfaces 12, 17185-17192. doi: 10.1021/acsami.9b23147

Menger, F. M. (1991). Groups of organic molecules that operate collectively. Angew. Chem. Int. Ed. 30, 1086-1099. doi: 10.1002/anie.199110861

Singh, N., Savanur, M. A., Srivastava, S., D’Silva, P., and Mugesh, G. (2017). A redox modulatory $\mathrm{Mn}_{3} \mathrm{O}_{4}$ nanozyme with multi-enzyme activity provides efficient cytoprotection to human cells in a Parkinson's disease model. Angew. Chem. Int. Ed. 56, 14267-14271. doi: 10.1002/anie.201708573

Song, Y. J., Qu, K. G., Zhao, C., Ren, J. S., and Qu, X. G. (2010). Graphene oxide: intrinsic peroxidase catalytic activity and its application to glucose detection. Adv. Mater. 22, 2206-2210. doi: 10.1002/adma.200903783

Sun, H. C., Miao, L., Li, J. X., Fu, S., An, G., Si, C. Y., et al. (2015). Self-assembly of cricoid proteins induced by "soft nanoparticles": an approach to design multienzyme cooperative antioxidative systems. ACS Nano 9, 5461-5469. doi: 10.1021/acsnano.5b01311

Wang, H., Wan, K. W., and Shi, X. H. (2019). Recent advances in nanozyme research. Adv. Mater. 31, 1805368. doi: 10.1002/adma.201805368

Wang, Q. B., Lei, J. P., Deng, S. Y., Zhang, L., and Ju, H. X. (2013). Graphenesupported ferric porphyrin as a peroxidase mimic for electrochemical DNA biosensing. Chem. Commun. 49, 916-918. doi: 10.1039/C2CC37664D

Wang, Y. X., Zhao, M. T., Ping, J. F., Chen, B., Cao, X. H., and Huang, Y., et al. (2016). Bioinspired design of ultrathin 2D bimetallic metal-organicframework nanosheets used as biomimeticenzymes. Adv. Mater. 28, 4149-4155. doi: 10.1002/adma.201600108

Wu, J. J., Wang, X. Y., Wang, Q., Lou, Z. P., Li, S. R., Zhu, Y. Y., et al. (2019). Nanomaterials with enzyme-like characteristics (nanozymes): next-generation artificial enzymes (II). Chem. Soc. Rev. 48, 1004-1076. doi: 10.1039/c8cs00457a

Xu, C., and Qu, X. G. (2014). Cerium oxide nanoparticle: a remarkably versatile rare earth nanomaterial for biological applications. NPG Asia Mater. 6:e90. doi: 10.1038/am.2013.88

Xu, C., Zhao, C. Q., Li, M., Wu, L., Ren, J. S., and Qu, X. G. (2014). Artificial evolution of graphene oxide chemzyme with enantioselectivity and nearinfrared photothermal effect for cascade biocatalysis reactions. Small 10, 1841-1847. doi: 10.1002/smll.201302750

Xue, T., Peng, B., Xue, M., Zhong, X., Chiu, C. Y., Yang, S., et al. (2014). Integration of molecular and enzymatic catalysts on graphene for biomimetic generation of antithrombotic species. Nat. Commun. 5, 3200-3205. doi: $10.1038 /$ ncomms 4200

Yin, W. Y., Yu, J., Lv, F. T., Yan, L., Zheng, L. R., Gu, Z. J., et al. (2016). Functionalized nano- $\mathrm{MoS}_{2}$ with peroxidase catalytic and nearinfrared photothermal activities for safe and synergetic wound antibacterial applications. ACS Nano 10, 11000-11011. doi: 10.1021/acsnano.6b05810

Zheng, B., Wang, C., Wu, C. Y., Zhou, X. J., Lin, M., Wu, X. C., et al. (2012). Nuclease activity and cytotoxicity enhancement of the DNA intercalators via graphene oxide. J. Phys. Chem. C 116, 15839-15846. doi: 10.1021/jp3050324

Zheng, B., Wang, C., Xin, X. Z., Liu, F., Zhou, X. J., Zhang, J. Y., et al. (2014). Electron transfer from graphene quantum dots to the copper complex enhances its nuclease activity. J. Phys. Chem. C 118, 7637-7642. doi: 10.1021/jp411348f

Conflict of Interest: The authors declare that the research was conducted in the absence of any commercial or financial relationships that could be construed as a potential conflict of interest.

Copyright (c) 2020 Huang, Yu, Qiu, Chu and Lin. This is an open-access article distributed under the terms of the Creative Commons Attribution License (CC BY). The use, distribution or reproduction in other forums is permitted, provided the original author(s) and the copyright owner(s) are credited and that the original publication in this journal is cited, in accordance with accepted academic practice. No use, distribution or reproduction is permitted which does not comply with these terms. 